\title{
Some Mobile Overconstrained Parallel Mechanisms
}

\author{
J.M. Selig
}

\begin{abstract}
The Griffis-Duffy platform is an example of an overconstrained parallel mechanism. Although it has 6 SS legs joining its platform to its base it is still mobile. In this work similar structures are found but with different types of legs. The key to finding these structures is a pair of theorems concerning 3 degree-of-freedom mechanisms subjected to a translation or a half-turn. Although these results are not new concise statements and proofs are given. These constructions are then applied to parallel mechanisms consisting of 3 RPS legs and 3UPU legs. Some details of the rigid-body motions that the platform of these mechanisms can execute are found. This is facilitated by the observations that rigid displacements permitted by an RPS leg are the displacements which constrain a point to a fixed plane, while the displacements of a UPU leg constrain a line to be coplanar to a fixed line.
\end{abstract}

Key words: Parallel mechanisms, overconstraint, line-symmetry.

\section{Introduction}

There has been much interest in overconstrained, single loop mechanisms such as the Bennett, Goldberg and various Bricard mechanisms. With interest turning to parallel mechanisms workers have also begun to look at over constrained parallel mechanisms. These are sometimes described as mechanisms which are architecturally singular. A key example of such a mechanism was the Griffis-Duffy platform as explained by Husty and Karger, [2]. Here these ideas are extended to platforms with other types of legs, in particular RPS and UPU. First we consider a pair of constructions which guarantee that the mechanisms will be mobile.

School of Engineering

London South Bank University, London SE1 0AA, U.K. e-mail: seligjm@1sbu.ac.uk 


\section{Line-Symmetry and Translations}

The results studied in this section are well known in general terms but giving a formal statement of the results clarifies the underlying geometry. In both cases the symmetries discussed confine the rigid motion to the intersection of a 5-dimensional projective space (a 5-plane) with the Study quadric.

In the following we will consider arbitrary mechanisms. These are to be thought of as systems of links and joints but we don't specify their arrangement so the mechanism could be a serial chain, a single loop or a parallel mechanism, for example. All we require is that one of the links be fixed and called the base link of the mechanism. We will concentrate our attention on another link in the mechanism and will refer to this as the coupler or platform of the mechanism. Saying that the coupler has 3-degrees-of-freedom then means that the possible displacements of the coupler, relative to the base link, can be specified using three parameters.

Lemma 1. Let $\mathscr{M}$ be an arbitrary mechanism having a coupler with 3 degrees-offreedom. Duplicate the mechanism $\mathscr{M}$ and subject the new one to a fixed translation. The translation must include all links and joints including the base link. After the translation the translated base-link is again fixed. Rigidly join the coupler bars of the two mechanisms to form a combined coupler. This combined coupler bar will be able to move and will, in general, follow a 1 degree-of-freedom Schönflies motion.

Proof. Assume that $g\left(\mu_{1}, \mu_{2}, \mu_{3}\right)$ is the dual quaternion representing the three parameter motion that the original mechanism $\mathscr{M}$ can perform. After a translation $t$, the shifted mechanism will be able to perform the motion, $\operatorname{tg}\left(\mu_{1}, \mu_{2}, \mu_{3}\right) t^{-}$, where $t^{-}$is the dual quaternion conjugate of $t$. When the couplers are joined together, any motion performed must satisfy,

$$
g\left(\mu_{1}, \mu_{2}, \mu_{3}\right)=\operatorname{tg}\left(\mu_{1}, \mu_{2}, \mu_{3}\right) t^{-} .
$$

This relation will have solutions for all displacements $g\left(\mu_{1}, \mu_{2}, \mu_{3}\right)$ that commute with $t$. The set of all elements in the group which commute with a translation consist of the subgroup of all translations and all rotations about axes parallel to $t$. That is, the centraliser of a translation is a Schönflies group. In the Study quadric a Schönflies group is the intersection of the Study quadric with a 5-plane. Intersecting with the 3-dimensional set of displacements $g\left(\mu_{1}, \mu_{2}, \mu_{3}\right)$ generally gives a 1-dimensional set, necessarily lying in the Schönflies subgroup.

Only the direction of the translation is important here, any translation in the same direction will give the same Schönflies group. For the parallel mechanisms considered below this means that the same motion can be generated by a machine with an arbitrary number of legs.

The second result is probably even more well known, the statement and simple proof are still instructive.

Lemma 2. Let $\mathscr{M}$ be an arbitrary mechanism that has a coupler with 3 degreesof-freedom. Again, duplicate the mechanism $\mathscr{M}$ but now subject the new one to a 
half-turn about a line $\ell_{0}$. This time the base-link of the new mechanism is rigidly fixed to the coupler link of the original and the coupler link of the new machine is fixed to the base. The coupler bar of the new combined mechanism will generally follow a 1 degree-of-freedom line-symmetric motion.

Proof. After the half-turn the motion of the coupler will be $\ell_{0} g\left(\mu_{1}, \mu_{2}, \mu_{3}\right) \ell_{0}^{-}$but the motion of the base with respect to the coupler will be, $\ell_{0} g^{-}\left(\mu_{1}, \mu_{2}, \mu_{3}\right) \ell_{0}^{-}$. After connecting the mechanism as specified the motion of the combined coupler will satisfy,

$$
g\left(\mu_{1}, \mu_{2}, \mu_{3}\right)=\ell_{0} g^{-}\left(\mu_{1}, \mu_{2}, \mu_{3}\right) \ell_{0}^{-} .
$$

This can be rearranged to produce,

$$
g\left(\mu_{1}, \mu_{2}, \mu_{3}\right) \ell_{0}^{-}+\ell_{0} g^{-}\left(\mu_{1}, \mu_{2}, \mu_{3}\right)=0,
$$

since $\ell_{0}^{-}=-\ell_{0}$. In [4] it was shown that this equation characterises line-symmetric motions, moreover, line-symmetric motions were shown to lie in the intersection of the Study quadric with a 5-plane.

In the following we look at 3-degree-of-freedom mechanisms formed by the parallel composition of three 5-degree-of-freedom serial chains or legs.

\section{RPS Legs}

The legs considered here are each composed of a revolute, prismatic and a final spherical joint, see Fig. 1. Keeping the base of the leg fixed and moving the platform attached to the final spherical joint gives a set of possible rigid-body displacements allowed by the leg. In all these displacements the centre of the spherical joint remains in contact with a fixed plane normal to the axis of the first revolute joint. Clearly, the set of displacements allowed by such a leg coincides with the pointplane constraint varieties discussed in [6] for example. These point-plane constraint varieties can be thought of as the intersection of the Study quadric in $\mathbb{P}^{7}$ with another quadric hypersurface.

\subsection{Schönflies 6RPS}

Here the construction of Lemma 1 is applied to a parallel mechanism consisting of three general RPS legs, see Fig. 3.

Assume the axis of the Schönflies motion is the $z$-axis so the rotation matrix and translation vector can be written

$$
R=\left(\begin{array}{ccc}
\cos \phi & -\sin \phi & 0 \\
\sin \phi & \cos \phi & 0 \\
0 & 0 & 1
\end{array}\right) \quad \text { and } \quad \mathbf{t}=\left(\begin{array}{c}
t_{x} \\
t_{y} \\
t_{z}
\end{array}\right)
$$




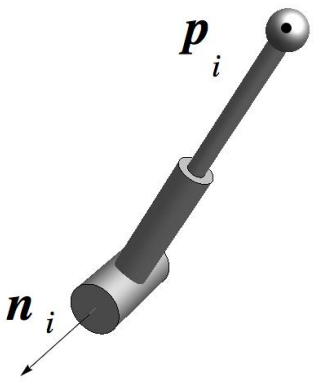

Fig. 1 An RPS Leg.

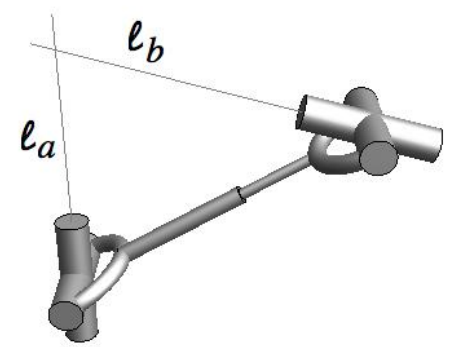

Fig. 2 A UPU Leg.

The three point-plane constraints can be written

$$
\left(\mathbf{n}_{i}^{T},-d_{i}\right)\left(\begin{array}{ll}
R & \mathbf{t} \\
0 & 1
\end{array}\right)\left(\begin{array}{c}
\mathbf{p}_{i} \\
1
\end{array}\right)=\mathbf{n}_{i}^{T}\left(R \mathbf{p}_{i}+\mathbf{t}\right)-d_{i}=0, \quad i=1,2,3,
$$

where $\mathbf{n}_{i}$ is the unit normal to the plane, $d_{i}$ the perpendicular distance from the plane to the origin and $\mathbf{p}_{i}$ the position vector of the point at the centre of the spherical joint. These equations can be written in matrix form as

$$
N \mathbf{t}=\boldsymbol{\delta}, \quad \text { where } \quad N=\left(\begin{array}{c}
\mathbf{n}_{1}^{T} \\
\mathbf{n}_{2}^{T} \\
\mathbf{n}_{3}^{T}
\end{array}\right) \quad \text { and } \quad \boldsymbol{\delta}=\left(\begin{array}{c}
d_{1}-\mathbf{n}_{1}^{T} R \mathbf{p}_{1} \\
d_{2}-\mathbf{n}_{2}^{T} R \mathbf{p}_{2} \\
d_{3}-\mathbf{n}_{3}^{T} R \mathbf{p}_{3}
\end{array}\right) .
$$

Assuming that the points $\mathbf{p}_{i}$ lie on their respective planes at the start of the motion, when $R(0)=I_{3}$, the row of $\boldsymbol{\delta}$ can be written $\mathbf{n}_{i}^{T}\left(I_{3}-R\right) \mathbf{p}_{i}$. Since $R$ determines a sequence of rotations about the $z$-axis, elements of $I_{3}-R$ can be written in terms of the sine and cosine of the rotation angle $\phi$.

The matrix $N$ can be inverted symbolically,

$$
N^{-1}=\frac{1}{\mathbf{n}_{1} \cdot\left(\mathbf{n}_{2} \times \mathbf{n}_{3}\right)}\left(\mathbf{n}_{2} \times \mathbf{n}_{3}\left|\mathbf{n}_{3} \times \mathbf{n}_{1}\right| \mathbf{n}_{1} \times \mathbf{n}_{2}\right) .
$$

So

$$
\mathbf{t}=N^{-1} \boldsymbol{\delta}=\boldsymbol{\alpha}(1-\cos \phi)+\boldsymbol{\beta} \sin \phi
$$

where

$$
\begin{aligned}
\boldsymbol{\alpha}=\frac{1}{\mathbf{n}_{1} \cdot\left(\mathbf{n}_{2} \times \mathbf{n}_{3}\right)}\left(\left(n_{1 x} p_{1 x}+n_{1 y} p_{1 y}\right) \mathbf{n}_{2} \times \mathbf{n}_{3}+\right. \\
\left.\left(n_{2 x} p_{2 x}+n_{2 y} p_{2 y}\right) \mathbf{n}_{3} \times \mathbf{n}_{1}+\left(n_{3 x} p_{3 x}+n_{3 y} p_{3 y}\right) \mathbf{n}_{1} \times \mathbf{n}_{2}\right)
\end{aligned}
$$

and 


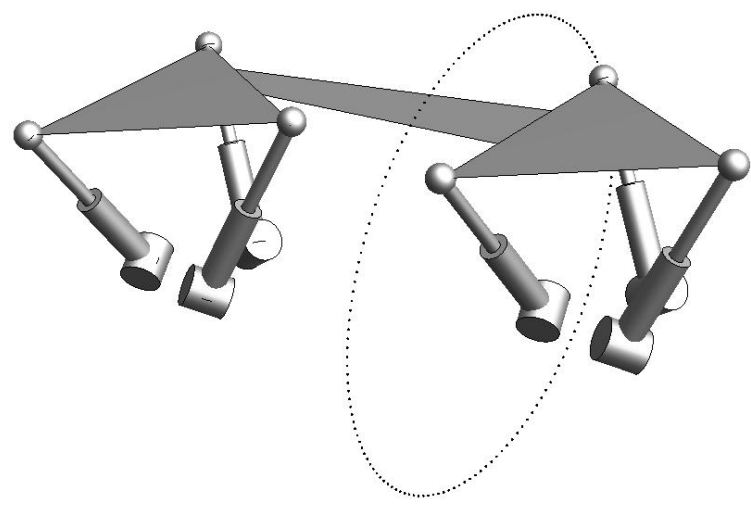

Fig. 3 A mobile 6RPS parallel mechanism constructed by translating a 3RPS linkage and joining the coupler bars. The dots represent the elliptical path of the centre of one of the spherical joints.

$$
\begin{aligned}
\boldsymbol{\beta}=\frac{1}{\mathbf{n}_{1} \cdot\left(\mathbf{n}_{2} \times \mathbf{n}_{3}\right)}\left(\left(n_{1 x} p_{1 y}-n_{1 y} p_{1 x}\right) \mathbf{n}_{2} \times \mathbf{n}_{3}+\right. \\
\left.\left(n_{2 x} p_{2 y}-n_{2 y} p_{2 x}\right) \mathbf{n}_{3} \times \mathbf{n}_{1}+\left(n_{3 x} p_{3 y}-n_{3 y} p_{3 x}\right) \mathbf{n}_{1} \times \mathbf{n}_{2}\right)
\end{aligned}
$$

Using the familiar tan-half-angle substitutions, $\cos \phi=\left(1-t^{2}\right) /\left(1+t^{2}\right)$ and $\sin \phi=$ $2 t /\left(1+t^{2}\right)$, it can be seen that the motion of an arbitrary point in the platform will be a conic curve in general. Hence this is a Darboux motion, see [1, Chap. IX, $\S 3]$. The fact that three point-plane constraints restricted to a Schönflies motion produces a Darboux motion is well known, see for example [8]. Notice however, that in [3] it was shown that a parallel mechanism with 3 RPS legs could perform a vertical Darboux motion, a particular Darboux motion that is also line-symmetric. The above shows that the mechanism can perform a general Darboux motion and indicates how to construct a mechanism to follow such a motion.

\subsection{Line-Symmetric 6RPS}

Next, the construction from Lemma 2 is applied to three RPS legs. As in the previous section there are three point-plane constraints given in equation (1).

Since a line-symmetric motion consists of successive half-turns about the generators of a ruled surface, the rigid-body displacements can be given by the exponential of a line

$$
\left(\begin{array}{ll}
R & \mathbf{t} \\
0 & 1
\end{array}\right)=e^{\pi L}, \quad \text { where } \quad L=\left(\begin{array}{cccc}
0 & -P_{03} & P_{02} & P_{23} \\
P_{03} & 0 & -P_{01} & P_{31} \\
-P_{02} & P_{01} & 0 & P_{12} \\
0 & 0 & 0 & 0
\end{array}\right)
$$


Here $P_{i j}$ are the Plücker coordinates of the line $L$. For a line-symmetric motion $L$ will be parametrised by time, however the explicit dependence on time has been suppressed for brevity. Using the Rodrigues formula, the exponential can be written:

$$
e^{\pi L}=I_{4}+2 L^{2},
$$

since $L^{3}=-L$ and assuming $P_{01}^{2}+P_{02}^{2}+P_{03}^{2}=1$. If we write

$$
\Omega=\left(\begin{array}{ccc}
0 & -P_{03} & P_{02} \\
P_{03} & 0 & -P_{01} \\
-P_{02} & P_{01} & 0
\end{array}\right), \quad \boldsymbol{\omega}=\left(\begin{array}{c}
P_{01} \\
P_{02} \\
P_{03}
\end{array}\right), \quad \text { and } \quad \mathbf{v}=\left(\begin{array}{c}
P_{23} \\
P_{31} \\
P_{12}
\end{array}\right),
$$

then the rotation matrix and translation vector can be written

$$
R=I+2 \Omega^{2}, \quad \mathbf{t}=2 \boldsymbol{\omega} \times \mathbf{v},
$$

where $\boldsymbol{\omega}$ and $\mathbf{v}$ consist of the Plücker coordinates of a line and so satisfy $\boldsymbol{\omega} \cdot \mathbf{v}=0$.

It is convenient to assume that the motion passes through the identity element of the group and that the points lie on their respective planes in this position. Then a line-symmetric motion is given by reflecting the three points $\mathbf{p}_{1}, \mathbf{p}_{2}$ and $\mathbf{p}_{3}$ in the initial line of the ruled surface $L_{0}$, and then reflecting in the successive lines of the surface, so that,

$$
\left(\begin{array}{ll}
R & \mathbf{t} \\
0 & 1
\end{array}\right)=\left(I_{4}+2 L^{2}\right)\left(I_{4}+2 L_{0}^{2}\right) \text {. }
$$

To be definite assume that the initial line $L_{0}$ is the $z$-axis. For simplicity, write $\mathbf{p}_{i}^{\prime}=$ $\left(I_{3}+2 \Omega_{0}^{2}\right) \mathbf{p}_{i}$, where $\Omega_{0}$ is the direction of the line $L_{0}$. The equations for the three point-plane constraints become, $\mathbf{n}_{\mathbf{i}}^{T}\left(I_{3}+2 \Omega^{2}\right) \mathbf{p}_{i}^{\prime}+2 \mathbf{n}_{\mathbf{i}}^{T}(\boldsymbol{\omega} \times \mathbf{v})-d_{i}=0$. Since $d_{i}=$ $\mathbf{n}_{\mathbf{i}}^{T}\left(I_{3}+2 \Omega_{0}^{2}\right) \mathbf{p}_{i}^{\prime}$, we get,

$$
\mathbf{n}_{\mathbf{i}}^{T}\left(\Omega^{2}-\Omega_{0}^{2}\right) \mathbf{p}_{i}^{\prime}+\mathbf{n}_{\mathbf{i}}^{T}(\boldsymbol{\omega} \times \mathbf{v})=0, \quad i=1,2,3 .
$$

These equations can be made homogeneous by multiplying the $\Omega_{0}^{2}$ term by the square of the norm of the vector $\boldsymbol{\omega}$, denoted $|\boldsymbol{\omega}|^{2}$. This results in three homogeneous equations,

$$
\mathbf{n}_{\mathbf{i}}^{T}\left(\Omega^{2}-|\boldsymbol{\omega}|^{2} \Omega_{0}^{2}\right) \mathbf{p}_{i}^{\prime}+\mathbf{n}_{\mathbf{i}}^{T}(\boldsymbol{\omega} \times \mathbf{v})=0, \quad i=1,2,3 .
$$

Including the equation for the Klein quadric $\boldsymbol{\omega} \cdot \mathbf{v}=0$, gives 4 homogeneous quadratic equations for the ruled surface generating the line-symmetric motion. The intersection of these quadrics is not a complete intersection as they clearly vanish on the 2-dimensional plane of "lines at infinity" $\boldsymbol{\omega}=\mathbf{0}$.

The equation given in (3) can be written in the same matrix vector form as in (2) but with

$$
\mathbf{t}=\boldsymbol{\omega} \times \mathbf{v} \quad \text { and } \quad \boldsymbol{\delta}=\left(\begin{array}{l}
\mathbf{n}_{1}^{T}\left(\Omega^{2}-|\boldsymbol{\omega}|^{2} \Omega_{0}^{2}\right) \mathbf{p}_{1}^{\prime} \\
\mathbf{n}_{2}^{T}\left(\Omega^{2}-|\boldsymbol{\omega}|^{2} \Omega_{0}^{2}\right) \mathbf{p}_{2}^{\prime} \\
\mathbf{n}_{3}^{T}\left(\Omega^{2}-|\boldsymbol{\omega}|^{2} \Omega_{0}^{2}\right) \mathbf{p}_{3}^{\prime}
\end{array}\right)
$$


The variables $\mathbf{v}$ can be eliminated by multiplying the equation $N \mathbf{t}=\boldsymbol{\delta}$, by the inverse or adjugate of $N$ and then taking the scalar product with $\boldsymbol{\omega}$. The result is

$$
\begin{aligned}
& \boldsymbol{\omega} \cdot\left(\mathbf{n}_{2} \times \mathbf{n}_{3}\right) \mathbf{n}_{1}^{T}\left(\Omega^{2}-|\boldsymbol{\omega}|^{2} \Omega_{0}^{2}\right) \mathbf{p}_{1}^{\prime}+\boldsymbol{\omega} \cdot\left(\mathbf{n}_{3} \times \mathbf{n}_{1}\right) \mathbf{n}_{2}^{T}\left(\Omega^{2}-|\boldsymbol{\omega}|^{2} \Omega_{0}^{2}\right) \mathbf{p}_{2}^{\prime}+ \\
& \boldsymbol{\omega} \cdot\left(\mathbf{n}_{1} \times \mathbf{n}_{2}\right) \mathbf{n}_{3}^{T}\left(\Omega^{2}-|\boldsymbol{\omega}|^{2} \Omega_{0}^{2}\right) \mathbf{p}_{3}^{\prime}=0 .
\end{aligned}
$$

This equation determines a plane cubic curve in the plane determined by the variables $P_{01}, P_{02}$ and $P_{03}$. This is the direction cone or spherical indicatrix of the generating surface of the motion. Generally a plane cubic curve is rational or elliptic (has genus 0 or 1) depending on whether or not it has a singularity. Computing with a few random examples shows that the curve can be non-singular. Hence, in general, the curve is elliptic. However, there may be particular examples where the cubic acquires a singularity and hence becomes rational.

Eliminating the moment vector $\mathbf{v}$ of the Plücker coordinates can be seen as a linear projection with centre of projection given by the 2-plane of lines "at infinity" $\boldsymbol{\omega}=\mathbf{0}$. To recover the moments of the generators in the ruled surface we can multiply the equation $N \mathbf{t}=\boldsymbol{\delta}$, by the inverse of $N$ and then take the vector product with $\boldsymbol{\omega}$ to get,

$$
\begin{aligned}
|\boldsymbol{\omega}|^{2} \mathbf{v} & =\frac{1}{\mathbf{n}_{1} \cdot\left(\mathbf{n}_{2} \times \mathbf{n}_{3}\right)}\left(\boldsymbol{\omega} \times\left(\mathbf{n}_{2} \times \mathbf{n}_{3}\right) \mathbf{n}_{1}^{T}\left(\Omega^{2}-|\boldsymbol{\omega}|^{2} \Omega_{0}^{2}\right) \mathbf{p}_{1}^{\prime}+\right. \\
\boldsymbol{\omega} & \left.\times\left(\mathbf{n}_{3} \times \mathbf{n}_{1}\right) \mathbf{n}_{2}^{T}\left(\Omega^{2}-|\boldsymbol{\omega}|^{2} \Omega_{0}^{2}\right) \mathbf{p}_{2}^{\prime}+\boldsymbol{\omega} \times\left(\mathbf{n}_{1} \times \mathbf{n}_{2}\right) \mathbf{n}_{3}^{T}\left(\Omega^{2}-|\boldsymbol{\omega}|^{2} \Omega_{0}^{2}\right) \mathbf{p}_{3}^{\prime}\right) .
\end{aligned}
$$

The equation for the Klein quadric has been used here to expand the triple product $\boldsymbol{\omega} \times(\boldsymbol{\omega} \times \mathbf{v})$. The above give a rational cubic map from the plane with homogeneous coordinates $\boldsymbol{\omega}=\left(P_{01}: P_{02}: P_{03}\right)$ to the Klein quadric. The image of the cubic spherical indicatrix will be a degree 9 ruled surface which meets the center of the linear projection, the space of lines at infinity, with multiplicity 6.

\section{6UPU Legs}

Here we study parallel mechanism composed of 6 UPU legs with either line symmetry or a translational symmetry. We require that the axes of the first and last revolute joints of the two U joints are coplanar, see Fig. 2. This is not the most general configuration for such a leg but it is a design that is commonly used in practice. The key observation is that the rigid displacement allowed by such a UPU leg will maintain the coplanarity of these lines. In [7] the problem of finding the set of rigid displacements which move a line in such a way that it remains in a linear line complex was studied. The set of lines meeting or parallel to a fixed line form a special linear line complex so the displacements of a UPU leg are a special case of the quadratic constraint found in [7]. That is, the displacements achievable by the leg lie on the intersection of the Study quadric with another quadric hypersurface in $\mathbb{P}^{7}$. There 


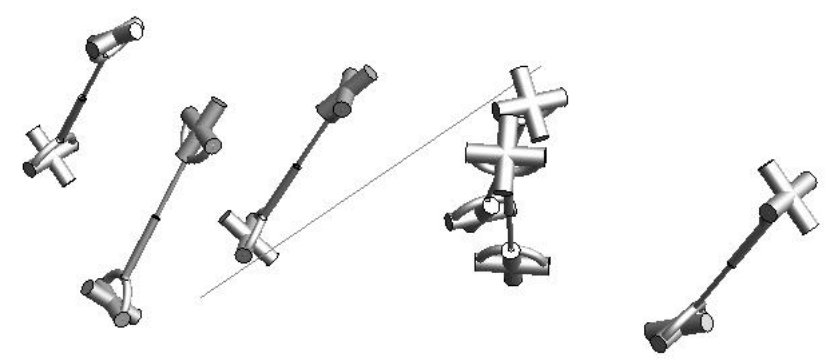

Fig. 4 A mobile 6UPU parallel mechanism constructed by reflecting a 3UPU linkage in the line shown. For clarity, the base and platform are not shown, the base is joined to the lower $\mathrm{R}$ joint of each leg and the platform to the uppermost $\mathrm{R}$ joints.

are several serial kinematic chains with the same property, the UPU chain, the PSP chain and RRPC are three examples. The remarks below therefore apply to any of these chains.

It is convenient here to represent rigid displacements using the adjoint representation of the group $S E(3)$. Consider a pair of lines given in terms of Plücker coordinates as,

$$
\ell_{a}=\left(\begin{array}{c}
\boldsymbol{\omega}_{a} \\
\mathbf{v}_{a}
\end{array}\right), \quad \text { and } \quad \ell_{b}=\left(\begin{array}{c}
\boldsymbol{\omega}_{b} \\
\mathbf{v}_{b}
\end{array}\right) .
$$

These lines will be coplanar if and only if they are reciprocal, this condition can be represented by the matrix equation,

$$
\left(\boldsymbol{\omega}_{a}^{T}, \mathbf{v}_{a}^{T}\right)\left(\begin{array}{cc}
0 & I_{3} \\
I_{3} & 0
\end{array}\right)\left(\begin{array}{c}
\boldsymbol{\omega}_{b} \\
\mathbf{v}_{b}
\end{array}\right)=0
$$

where $I_{3}$ is the $3 \times 3$ identity matrix. The rigid displacement which move $\ell_{b}$ in such a way that it remains coplanar to $\ell_{a}$ will thus satisfy the equation

$$
\left(\boldsymbol{\omega}_{a}^{T}, \mathbf{v}_{a}^{T}\right)\left(\begin{array}{cc}
0 & I_{3} \\
I_{3} & 0
\end{array}\right)\left(\begin{array}{cc}
R & 0 \\
T R & R
\end{array}\right)\left(\begin{array}{c}
\boldsymbol{\omega}_{b} \\
\mathbf{v}_{b}
\end{array}\right)=0
$$

where $R$ is a rotation as above and $T$ is the translation vector written as a $3 \times 3$ anti-symmetric matrix. Expanding the equation above produces

$$
\boldsymbol{\omega}_{a}^{T} T R \boldsymbol{\omega}_{b}+\boldsymbol{\omega}_{a}^{T} R \mathbf{v}_{b}+\mathbf{v}_{a}^{T} R \boldsymbol{\omega}_{b}=0 .
$$

Three such legs yield an equation of the form $N \mathbf{t}=\boldsymbol{\delta}$ again. This time with

$$
N=\left(\begin{array}{c}
\left(\boldsymbol{\omega}_{a 1} \times\left(R \boldsymbol{\omega}_{b 1}\right)\right)^{T} \\
\left(\boldsymbol{\omega}_{a 2} \times\left(R \boldsymbol{\omega}_{b 2}\right)\right)^{T} \\
\left(\boldsymbol{\omega}_{a 3} \times\left(R \boldsymbol{\omega}_{b 3}\right)\right)^{T}
\end{array}\right) \quad \text { and } \quad \boldsymbol{\delta}=\left(\begin{array}{c}
\boldsymbol{\omega}_{a 1}^{T} R \mathbf{v}_{b 1}+\mathbf{v}_{a 1}^{T} R \boldsymbol{\omega}_{b 1} \\
\boldsymbol{\omega}_{a 2}^{T} R \mathbf{v}_{b 2}+\mathbf{v}_{a 2}^{T} R \boldsymbol{\omega}_{b 2} \\
\boldsymbol{\omega}_{a 3}^{T} R \mathbf{v}_{b 3}+\mathbf{v}_{a 3}^{T} R \boldsymbol{\omega}_{b 3}
\end{array}\right)
$$


Notice that the matrix $N$ depends on the rotation $R$ now. As above, duplicating and translating the three legs produces a mechanism which can perform a Schönflies motion. Assuming that the rotation is parametrised by quadratic functions, as in section 3.1, we can solve for the translation $\mathbf{t}$. This will give a solution of degree 6 in the tan-half angle of the rotation. In particular the trajectories of general points on the platform of the mechanism will follow rational curves of degree 6 .

Finally consider duplicating the legs and subjecting them to a half-turn, as in section 3.2, see also Fig. 4. The spherical indicatrix of the base surface of the linesymmetric motion will be a planar curve of degree 7 . From the genus-degree formula the maximum genus of such a curve is $\frac{1}{2}(7-1)(7-2)=15$.

\section{Conclusion}

Space restrictions preclude a fuller discussion of the motions generated by these mechanisms. Although much is known about motions which constrain points to planes and to spheres, the case of motion in which lines remain coplanar to fixed lines seems to have not received much attention to date.

\section{References}

1. O. Bottema and B. Roth, 1990, Theoretical Kinematics, Dover Publications, New York.

2. M.L. Husty and A. Karger, 2000,"Self-motions of Griffis-Duffy type parallel manipulators," Proc. ICRA '00, vol 1, pp. 7-12, IEEE Int. Conf. on Robotics and Automation, San Francisco, CA.

3. L. Nurahmi, J. Schadlbauer, M. Husty, P. Wenger and S, Caro, 2014, "Motion capabilities of the 3-RPS cube parallel manipulator.", pp. 527-535 in Advances in Robot Kinematics eds. J. Lenarčič and O. Khatib, Springer International Publishing, Switzerland.

4. J.M. Selig and M. Husty, 2011, "Half-turns and line symmetric motions", Mechanism and Machine Theory, 2011, 46(2):156-167.

5. J.M. Selig,2005, Geometric Fundamentals of Robotics, Springer Verlag, New York.

6. J.M. Selig, 2011, "On the geometry of point-plane constraints on rigid-body displacements", Acta Applicandae Mathematicae, 116(2):133-155.

7. J.M. Selig, 2013, "On the geometry of the homogeneous representation for the group of proper rigidbody displacements", Romanian Journal of Technical Sciences - Applied Mechanics, (Special issue on New Trends in Advanced Robotics), 58(1-2):27-50.

8. Zsombor-Murray, P.J. and Gfrerrer, A., 2010, "A unified approach to direct kinematics of some reduced motion parallel manipulators" ASME Journal of Mechanisms and Robotics, 2(2):021006 (10 pages). 